ESTIMATION OF THE CRESSMAN TERM FOR A

BAROTROPIC MODEL THROUGH OPTIMIZATION WITH USE OF THE ADJOINT MODEL

Rinne, Juhani

1993-03

Rinne , J \& Järvinen , H 1993 , ' ESTIMATION OF THE CRESSMAN TERM FOR A

BAROTROPIC MODEL THROUGH OPTIMIZATION WITH USE OF THE ADJOINT MODEL

' , Monthly weather review , vol. 121 , no. 3 , pp. 825-833 . https://doi.org/10.1175/1520-0493(1993)121<0825:EOTC

http://hdl.handle.net/10138/313524

https://doi.org/10.1175/1520-0493(1993)121<0825:EOTCTF>2.0.CO;2

publishedVersion

Downloaded from Helda, University of Helsinki institutional repository.

This is an electronic reprint of the original article.

This reprint may differ from the original in pagination and typographic detail.

Please cite the original version. 


\title{
Estimation of the Cressman Term for a Barotropic Model through Optimization with Use of the Adjoint Model
}

\author{
JUHANI RINNE AND HEIKKI JÄRVINEN \\ Department of Meteorology, University of Helsinki, Hallituskatu, Helsinki, Finland
}

(Manuscript received 17 April 1991, in final form 17 July 1992)

ABSTRACT

\begin{abstract}
This paper presents an estimate of the Cressman term that appears in the divergent barotropic model as a corrector of planetary-wave retrogression. In contrast with earlier studies, the term varies as a function of geographical location. The estimation is performed using the adjoint model.

The results deviate from those earlier derived theoretically and applied in routine forecasting. The Cressman term can now be viewed as a corrector of the systematic error or as a baroclinity parameter. The proposed form of the Cressman term can also be interpreted as a forcing parameter, maintaining the troughs and ridges of the main circulation by affecting the free long waves.

Parameter estimation using the adjoint model has shown its potential in these experiments. Not only are the parameter values determined but new interpretations and approaches have been found.
\end{abstract}

\section{Introduction}

From the point of view of weather forecasting, the most remarkable step in numerical meteorology has obviously been the introduction of the barotropic model. With it, forecasting work was suddenly brought into the numerical era. The model was used widely in various forecasting centers. Besides the computational development, the only major change later made to the model was the addition of the so-called Cressman term (Cressman 1958). With this change, the nondivergent barotropic model was replaced by the divergent one.

The change improved the forecasts considerably. Cressman ends his paper by remarking, "It has frequently been stated that numerical forecasts cannot be considered to be in competition with other forecast systems . . . The above results . . . suggest that such statements . . . can no longer be considered valid!"

Since the model was used so widely and the improvement was clear, the Cressman term underwent thorough testing. One might expect that an optimal parameterization of the term was found. This has, however, never been verified.

In this paper, our aim is to find the optimal value of the Cressman term by means of the adjoint model (Talagrand and Courtier 1987; Courtier and Talagrand 1987). Traditionally, the value of the Cressman term has been treated as a constant. It has thus been simply a matter of trial and error to find the numerical value of the term that gives the best forecasts. In contrast

Corresponding author address: Juhani Rinne, University of Helsinki, Department of Meteorology, Hallituskatu 11-13, SF-00100 Helsinki, Finland. with that approach, we let the term vary as a function of geographical location. In practice, it is then no longer possible to estimate the value of the term by experimenting at every grid point, which is why the adjoint model is applied. We are interested to discover, in light of the results of a modern optimization method, how well the earlier theory and experimental development did actually perform. Interestingly enough, Wiin-Nielsen (1991) has recently described the early steps taken in numerical weather forecasting. Now seems to be an appropriate time to document and evaluate that phase of its development.

In addition to the historical approach, we have our own interest in using the adjoint model in parameter estimation. The barotropic model is a suitable tool for experimentation, even though it is no longer in operational use. Accordingly, we pose two questions. First, What in fact would have been the optimal value for the Cressman term? Second, How can that question be answered with the use of the adjoint equations?

In this paper, we use the specific historical nameCressman term-that was once generally employed. The term has been so important that a specific name is indeed justified. "Bolin-Cressman term," however, would obviously be more correct (cf. Cressman 1958; Wiin-Nielsen 1991).

\section{The data and the model}

In this study, analyses of the 500-hPa geopotential field are used, which are given on the National Meteorological Center (NMC) polar stereographic grid (grid interval $381 \mathrm{~km}$ at $60^{\circ} \mathrm{N}$ ), at a total of 1404 points north of $20^{\circ} \mathrm{N}$. 
The barotropic forecasting model for the streamfunction $\psi$ reads

$$
\left(\nabla^{2}-\frac{q}{\mu}\right) \frac{\partial \psi}{\partial t}=J\left(\mu \nabla^{2} \psi+f, \psi\right),
$$

where $\nabla^{2}$ and $J$ stand for the numerical Laplacian and Jacobian operators [given, for our case, later in (4a) and (4c)]. Further, $q$ and $\mu$ are, respectively, the Cressman parameter and the map factor related to the grid used, and $f$ is the Coriolis term. For the balance equation, $\psi=\left(g / f_{0}\right) z$, where $g$ and $z$ refer to gravity and the $500-\mathrm{hPa}$ geopotential height, respectively, and $f_{0}$ is the fixed value of $f$ at latitude $60^{\circ} \mathrm{N}$. The tangent linear equation of (1a) is (Talagrand and Courtier 1987)

$$
\left(\nabla^{2}-\frac{q}{\mu}\right) \frac{\partial}{\partial t} \delta \psi=J\left(\mu \nabla^{2} \delta \psi, \psi\right)+J\left(\mu \nabla^{2} \psi+f, \delta \psi\right) .
$$

The adjoint model of ( $\mathrm{lb}$ ) is developed following Talagrand and Courtier (1987) using the "meteorological" approach, although the "oceanographic" approach applying Lagrange multipliers is much easier to handle and understand (cf. Thacker 1988). The main difference between our case and that of Talagrand and Courtier is that, instead of a spectral approach, a grid that is given in a quasi-circular area of 1404 points is used. Thus, a grid model is applied, as was the case in Cressman's time.

To develop the adjoint model corresponding to (1b), an inner product in Hilbert space is required. This will be defined as follows:

$$
\left\langle\zeta, \zeta^{\prime}\right\rangle=-\sum_{A} \psi\left(\nabla^{2}-\frac{q}{\mu}\right) \psi^{\prime} .
$$

Here, $\zeta=\nabla^{2} \psi$, and the summation is taken over the grid. For $q>0,(2)$ is positive. It may become negative when $q<0$. Nevertheless, (2) has been applied. In the $220+200$ forecasts run in this work, the formulas derived performed well, even though locally $q<0$.

The formulas and their derivation are similar to those of the spectral approach, so only the results are presented here. The Appendix, however, shows that our adjoint equation really is the adjoint of the tangent linear equation in its numerical form (1b).

The adjoint counterpart of the forecasting model [( $1 \mathrm{~b})$ when first discretized with respect to time, applying the leapfrog scheme] reads

$$
\begin{aligned}
\left(\nabla^{2}-\frac{q}{\mu}\right) \Delta\left(\delta \psi^{\prime}\right)=-2 & \Delta t\left\{-J^{*}\left(\delta \psi_{n+1}^{\prime}, \mu \nabla^{2} \psi_{n}+f\right)\right. \\
+ & \left.\nabla^{2}\left[\mu J^{*}\left(\delta \psi_{n+1}^{\prime}, \psi_{n}\right)\right]\right\} .
\end{aligned}
$$

In (3), $J^{*}$ denotes the adjoint counterpart of the numerical Jacobian operator $J ; \delta \psi^{\prime}\left[\Delta\left(\delta \psi^{\prime}\right)=\delta \psi_{n+2}^{\prime}\right.$ $\left.-\delta \psi_{n}^{\prime}\right]$ is the variable to be predicted by the adjoint model. The subscript $n$ refers to the corresponding time step of the barotropic model. The adjoint leapfrog tirne stepping is similar to that of the spectral model (cf. Appendix in Courtier and Talagrand 1987).

The Jacobian occurring in models (1a) and ( $1 \mathrm{~b})$ is computed at an inner grid point as follows:

$$
\begin{aligned}
J(a, b)= & \frac{1}{4}\left[\left(a_{i+1, j}-a_{i-1, j}\right)\left(b_{i, j+1}-b_{i, j-1}\right)\right. \\
& \left.-\left(a_{i, j+1}-a_{i, j-1}\right)\left(b_{i+1, j}-b_{i-1, j}\right)\right] .
\end{aligned}
$$

Here $a$ and $b$ are variables and $(i, j)$ refers to the grid point. The corresponding indexing in the adjoint model (3) becomes

$$
\begin{aligned}
J^{*}(a, b)= & \frac{1}{4}\left[a_{i+1, j}\left(b_{i+1, j+1}-b_{i+1, j-1}\right)\right. \\
& \quad-a_{i-1, j}\left(b_{i-1, j+1}-b_{i-1, j-1}\right) \\
& -a_{i, j+1}\left(b_{i+1, j+1}-b_{i-1, j+1}\right) \\
& \left.\quad+a_{i, j-1}\left(b_{i+1, j-1}-b_{i-1, j-1}\right)\right] .
\end{aligned}
$$

The Laplacian is the traditional one:

$$
\nabla^{2} a_{i, j}=a_{i+1, j}+a_{i-1, j}+a_{i, j+1}+a_{i, j-1}-4 a_{i, j} \text {. }
$$

At the boundaries, the right-hand sides of (4a) and (4b) are set to zero, and instead of (4c), a three-point formula is applied. There are some exceptions at the corner points. In assimilation experiments, very weak, fast-moving waves, which grow rapidly after reaching the boundaries, are observed. In the model forecasts, the waves were long, whereas in the adjoint stage they appeared as short waves, as those of Amodei and Bernardet (1990). Here these waves will not be discussed, as in this work they do not appear because we do not assimilate but rather estimate the value of one parameter. For the same reason, the boundary conditions can be easily chosen. We take the boundary values, separately for each time step, directly from analyses by interpolating between successive analyses. Otherwise the boundary conditions are those normally applied with the barotropic model (no model-induced change at the boundaries). This holds true for the adjoint model, too.

The penalty function $P$ is given by

$$
P=\sum_{A}\left(z_{\text {for }}-z_{\text {ver }}\right)^{2} d A,
$$

where $z_{\text {for }}$ and $z_{\text {ver }}$ refer to the 24-h forecast and verification analyses, respectively. The corresponding term must be added on the right-hand side of ( 3 ) as is done in the spectral model (cf. Appendix in Courtier and Talagrand 1987). The area elements $d A$ are given separately at each grid point and are determined so that their sum over the grid equals unity.

The aim of the adjoint computations is to determine $\partial P / \partial q$. This is computed by determining

$$
\left(\frac{\partial P}{\partial q}\right)_{n}=\delta \psi_{n}^{\prime}\left(\psi_{n}-\psi_{n-2}\right) \frac{1}{\mu}
$$


during each time step at each grid point (with minor exceptions for small values of $n$ or the first forecast steps). These values are then summed over the integration period. The derivative is thus given at each grid point separately.

The computations proceed in a normal manner where a 24-h barotropic forecast is followed by the backward integration of the adjoint model. The derivative of the penalty function, the result of the computations, is a field that tells separately at each grid point how much a small increment in $q$ changes the value of the penalty function. To minimize the penalty (5), a better approximation to the Cressman term can thus be found through

$$
q_{\text {new }}=q-\rho \frac{\partial P}{\partial \mathrm{q}} .
$$

The procedure is then repeated with this new value. If the values of $\rho$ are suitably chosen, the process converges with repeated scans, and a $q$ field corresponding to the minimum of the forecasting error is found. Note that $q$ is separately determined at each grid point. The values of $\rho$ were chosen experimentally since the present computations do not require much computing time, and there was thus no specific need for an optimization of the convergence.

As the penalty function is nonlinear, there may be several local minima. This was checked by using different first guesses and by applying different values of $\rho$. The computations always resulted in similar solutions, however. An exception is described in section 5.

\section{What is expected for the Cressman term?}

Wolff (1958) showed that forecasts made by a nondivergent barotropic model were improved considerably by forcing the long waves to be unchanged. Cressman (1958) goes back to Rossby et al. (1939) when pointing out that the phase velocity of divergent long waves is slower than that of nondivergent ones. Instead of locking the long waves, Cressman, in applying a two-layer model, looked for a correction term in the model. He found that

$$
q=\frac{f \eta}{(1-\epsilon) g h} .
$$

Here $\eta$ and $h$ are the absolute vorticity of the lower fluid layer and the height of the interface, respectively. The factor $\epsilon$ is given by the ratio of the density of the upper fluid to that of the lower fluid. Cressman stated that the evaluation of $\epsilon$ is doubtful and decided to find a suitable value of $q$ by experimenting with the model. This approach was then applied in the operational models.

Cressman studied a sample of ten forecasts. The best choice, as interpreted by Döös (1969), turned out to be $q=0.75 \times 10^{-12} \mathrm{~m}^{-2}$. In the following, this value will be denoted as $q_{0}$. According to Cressman's results, $1.5 \times 10^{-12} \mathrm{~m}^{-2}$, that is, $2 q_{0}$, could be used just as well. This corresponds to the value presented by Bolin (1956). The values for the Cressman and Bolin cases given in Wiin-Nielsen (1991) correspond to $0.93 q_{0}$ and $1.33 q_{0}$, respectively.

The same term occurs in Haltiner and Williams (1980), derived for an integrated model. The integration of the divergence term results in $q=f^{2} A_{0} / R T_{0}$. Here $A_{0}$ gives the relation between the surface and upper winds, $R$ refers to the gas constant, and $T_{0}$ refers to surface temperature. If $A_{0}=0.1$ and $f=10^{-4} \mathrm{~s}^{-1}$, then $q=0.016 q_{0}$ (exceptionally small).

A straightforward approach results if one considers the conservation of potential vorticity and approximates the term due to the divergence by $-q z$ (Holton 1979). Then

$$
-q z=f_{0}^{2} \frac{\partial}{\partial p}\left(\frac{1}{\sigma} \frac{\partial z}{\partial p}\right)
$$

where $\sigma$ is the stability parameter and partial derivatives are taken with respect to pressure. Holton gives $q$ $\approx 1.3 q_{0}$. The assumption made about the vertical structure is directly visible. If the stability parameter $\sigma$ is constant, then $q$ is positive for wavelike vertical structures. Let the phase shift in a strongly baroclinic layer be $65^{\circ}$. If the thickness of the layer is $500 \mathrm{hPa}$ and $\sigma=2 \times 10^{-6} \mathrm{~m}^{2} \mathrm{~Pa}^{-2} \mathrm{~s}^{-2}$, then $q$ becomes as large as $3.4 q_{0}$. Negative values result if $z$ exponentially decreases or increases. This can happen for easterly winds (Charney 1973).

If the model is understood to describe shallow-water conditions, then the Cressman term is related to the square of the Rossby radius of deformation. Accordingly, the term must always be positive. Both Holton and Wiin-Nielsen follow this approach, since their symbol for the term is a squared 1 , indicating that the term is always positive.

The most recent value given for the term, $2.11 q_{0}$, is that derived by Wiin-Nielsen (1991) in his historical review.

The Cressman term was thus intended to parameterize the divergence associated with the long waves. In most of the theoretical approaches, the term is related to $f \eta$ or $f^{2}$. In addition to this, it can be related to a structural parameter of a two-layer model, the inverse of the height, the surface wind, the vertical wavelength, or the Rossby radius of deformation. In practice, the term was not related to $f^{2}$ but was approximated by a constant, the optimum value of which was unknown. A suitable value is $q_{0}$, but larger values could be used just as well. Negative values could be considered either in exceptional cases only or not at all. In this paper, $q_{0}$ will be used as the basic comparison value.

Because the boundary values are taken from analyses, they do not depend on the model or on $q$. Thus, $q$ can be arbitrarily chosen at the boundaries. Different 
choices were experimented with, but these did not essentially affect the results at inner points. Therefore, $q$ $=q_{0}$ was chosen at the boundaries.

\section{What was found for the Cressman term?}

Our studies are based on a sample of 220 NMC analyses that were used as initial conditions. These were taken from the winter months of December, January, and February of 1965-75 at 4-day intervals. The analyses should thus be rather independent of each other.

Before applying the adjoint model for estimation, our own classical value for the Cressman term was determined. We experimented with the analysis sample and tried, by performing several 24-h forecasts of the 220 cases with different numerical values of $q$, to find the value that results in the best forecasts as measured with the penalty function. At this stage, the Cressman term was constant over the whole grid and the best value, in this sample, turned out to be $1.3 q_{0}$. The penalty function is very flat, however, and values of $1.0 q_{0}$ and $1.8 q_{0}$ could just as well be used. The result depends very much on the penalty function. If one wishes to minimize the systematic error, then $q$ should be larger than $5 q_{0}$. For a minimum of the random error, however, the optimum value is close to $0.7 q_{0}$. These two factors, the flatness of the penalty function and the choice of the variable to be minimized, explain the large variation in the historical values; any of them could have been used.

We next turned to the adjoint computations and did not consider the Cressman term as a single constant

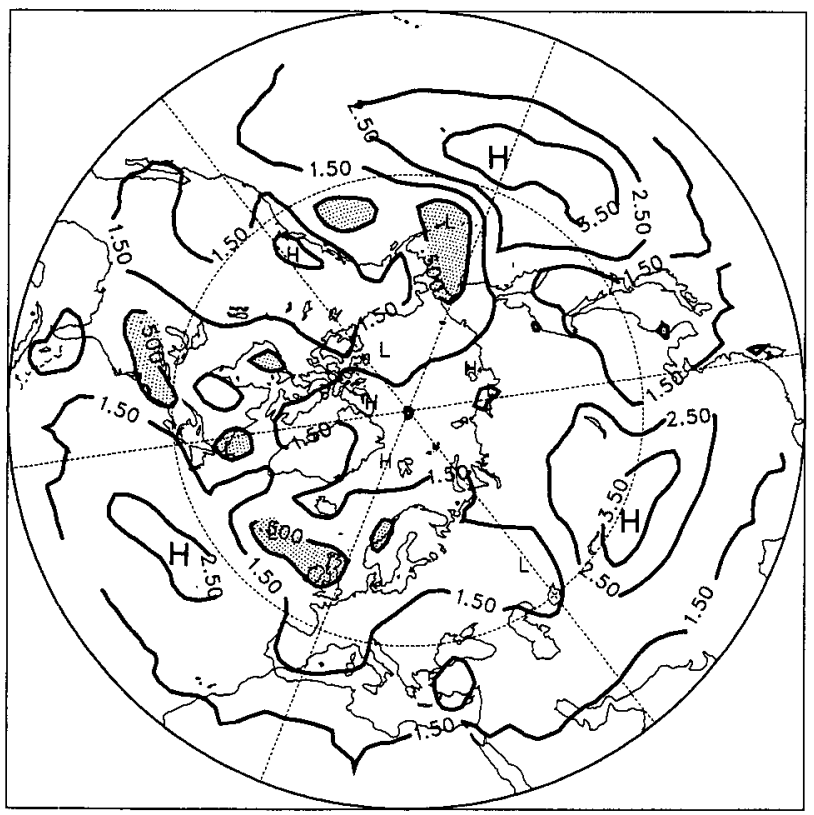

FIG. 1. Solution of the adjoint computations. The values of $q$ are given in relative terms of $q / q_{0}$. The maximum and minimum values are $q=4.4 q_{0}$ and $q=-0.5 q_{0}$. Areas where $q<0.5 q_{0}$ are shaded.
TABLE 1. Height forecast errors in 220 (200) winter cases of dependent (independent) data for various values of parameter $q$, given in meters.

\begin{tabular}{|c|c|c|c|}
\hline$q$ & Total rmse & Systematic error & Random rmse \\
\hline \multicolumn{4}{|c|}{ Dependent data } \\
\hline 0 & 81.8 & 47.8 & 66.3 \\
\hline$q_{0}$ & 61.6 & 20.4 & 58.2 \\
\hline As in Fig. 1 & 59.6 & 15.1 & 57.6 \\
\hline As in Fig. 3 & 58.7 & 11.4 & 57.5 \\
\hline \multicolumn{4}{|c|}{ Independent data } \\
\hline 0 & 79.3 & 48.7 & 62.6 \\
\hline$q_{0}$ & 57.4 & 19.5 & 54.0 \\
\hline As in Fig. 1 & 55.7 & 15.0 & 53.7 \\
\hline As in Fig. 3 & 54.5 & 11.3 & 53.3 \\
\hline
\end{tabular}

any longer. Better estimates of $q$ were repeatedly determined, starting with an overall constant value of $q_{0}$ $=0.75 \times 10^{-12} \mathrm{~m}^{-2}$. In these computations, the analyses were not changed in any way.

We first experimented with a single initial analysis. The value of $q$ was gradually changed at each inner grid point separately, while the forecast error simultaneously decreased. In fact, the computations can be seen as a mapping from the forecast error of the particular case onto the Cressman parameter. The computations converge toward a certain $q$ field. In this case, the corresponding forecast error will be equal to zero because there are as many $q$ values to be determined as there are inner grid points. Note that the penalty function equals zero at the boundary points and there is thus nothing to optimize because the boundary values are interpolated from the analyses.

To find a more universal solution, we next studied our sample of 220 initial conditions. At each forecastadjoint step, $\partial P / \partial q$ was determined separately for each initial condition. The sum of derivatives was then computed at each grid point in order to find the final derivative field. It took about 12 iteration steps to find a suitable solution (Fig. 1). Note, however, that we did not try to optimize the computations and that $\rho$ was chosen experimentally.

To describe the results, the following terms were computed for Table 1:

$$
\begin{aligned}
& \text { total rmse }=\left[\frac{1}{T} \sum^{N} \sum^{T}\left(z_{\mathrm{for}}-z_{\mathrm{ver}}\right)^{2} d A\right]^{1 / 2} \\
& \text { systematic error }=\frac{1}{T} \sum^{N} \sum^{T}\left(z_{\mathrm{for}}-z_{\mathrm{ver}}\right) d A
\end{aligned}
$$

random rmse

$$
=\left[(\text { total rmse })^{2}-(\text { systematic error })^{2}\right]^{1 / 2} \text {. }
$$

Here $T$ is the number of forecasts cases ( $=220$ ) and $N$ the number of grid points $(=1404)$, and $z_{\text {for }}$ and $z_{\text {ver }}$ 
are defined as in (5). Note that the total rmse is related to the root of the penalty function.

According to the results, the process converged. The total rmse, as verified against the NMC analyses, decreased because of a decrease in the systematic error (Table 1, second and third rows). The process can thus also be interpreted as a complex mapping from the systematic error onto the Cressman term.

Table 1 also shows how the Cressman term of Fig. 1 performs in a sample (called "independent data") that is not used in the estimation of the parameter. For this comparison, a set of $200 \mathrm{NMC}$ analyses was used from the winters of 1976-86 sampled at 3-day intervals.

During the first iterations, a long-wave structure developed in the $q$ field. The computations were interrupted when small-scale cells began to appear. It was thought that they were probably not significant. Simultaneously, the random error tended to increase, which, too, was taken as a reason for interruption.

When interpreting the result for 220 winter cases (Fig. 1), one can immediately see that the term is not correlated with the surface wind, nor does it increase with the square of the Coriolis term. Both of these parameters are large, for instance, during winter over Ireland and the Aleutians, where $q$ in the present results even takes negative values.

There are four maxima: over the western parts of the Pacific, over the Atlantic, over Siberia (or north of the Himalayas), and over North America (or close to the Rocky Mountains). In these areas, values close to or above $2 q_{0}$ are found, with a maximum of over $4.4 q_{0}$. Negative values, though, are found only locally. Note that the flatness of the field at the boundaries is due to the boundary assumptions made on $q$. The mean value of the field is $1.2 q_{0}$.

We study the term $(1 / T) \sum_{t}^{T}\left(z_{\mathrm{for}}-z_{\mathrm{ver}}\right)$, that is, the behavior of the systematic error in the grid points. This decreases throughout (Figs. 2a, b) when applying different $q$ values in (1a). The relation between the extrema in the systematic error and the maxima in $q$ can be seen by comparing Figs. 1 and 2 .

There is obviously no doubt about the significance of the results because the test on an independent dataset (Table 1) is very close to that for dependent data.

What would happen if the random error were penalized instead of the total one? Similar distribution of maxima and minima of $q$ are again found, although their intensities, sizes, and locations are somewhat different. These large-scale features, however, rapidly become noisy with more adjoint scans. If the result of the third scan is chosen, then both the total error and the systematic error are worse than in the initial case of $q=q_{0}$, whereas the random error is slightly better. Thus, the new $q$ field produces no significant improvement. The mean value of the field is $1.0 q_{0}$.

\section{Extended experiments}

In the model (1a), the Cressman term does not change the vorticity-tendency patterns but rather their intensities in such a way that the positive values of $q$ weaken the tendencies. The main difference between the barotropic and other models lies just in the barotropy, and one may thus suppose that the task of the
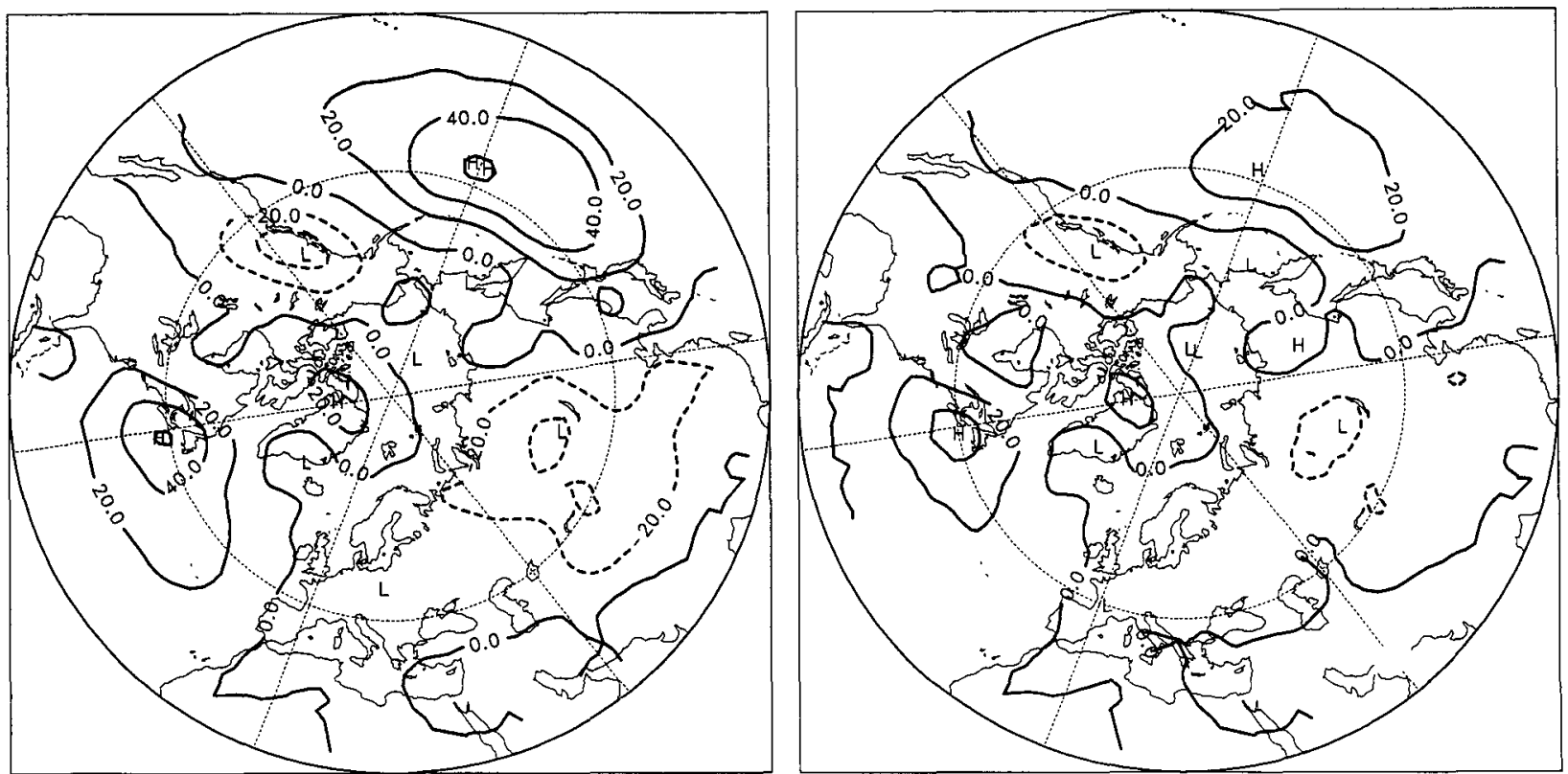

FIG. 2. The systematic forecasting error in the dependent data of 220 winter cases when applying the classical value of (a) $q_{0}$ and (b) that of Fig. 1 (b). The 500 -hPa height error is given in meters; negative isolines are dashed. 
Cressman term is to change tendencies where the barotropic tendencies systematically differ from the baroclinic ones. This is supported by the fact that the $q$ values are large over oceanic areas of strong baroclinity. All this tempts one to investigate whether $q$ values could be different in cases of positive and negative tendencies. Therefore, the model (1a) is changed accordingly:

$\left[\nabla^{2}-k \frac{q_{+}}{\mu}-(1-k) \frac{q_{-}}{\mu}\right] \frac{\partial \psi}{\partial t}=J\left(\mu \nabla^{2} \psi+f, \psi\right)$,

where $k=1$ for $\partial \psi / \partial t>0$ and $k=0$ for $\partial \psi / \partial t \leqslant 0$.

The application of a double-valued Cressman term in (11) requires one to know the sign of the tendency at the actual grid point. It was decided that the sign would be chosen as follows: because $\partial \psi / \partial t$ and the right-hand side of (11) are almost always of opposite sign, $k=1(k=0)$ is taken for rhs $<0$ (rhs $\geqslant 0)$.

The idea of a double-valued $q$ term is further supported by the systematic errors. The error fields were computed separately for positive and negative 24-h tendencies. The barotropic model should be incapable of deepening baroclinic systems sufficiently, and thus for negative tendencies, the systematic error is positive. In fact, the corresponding systematic error (not shown) is positive practically everywhere. Maxima are found over the Pacific and the Atlantic, where the systematic error exceeds $100 \mathrm{~m}$. Elsewhere the field is weak. On the other hand, for positive tendencies, the extrema of the systematic errors are found over North America and Siberia. Elsewhere the error field is weak but negative throughout, indicating that the model systematically underestimates the increasing tendencies. Thus, counterparts of the four maxima of the $q$ field in Fig. 1 are found in the maps of systematic errors associated with different tendency signs.

The computations converged. In this case there was no need to interrupt them because of the appearance of small scales, as was so in the previous case. After eight scans, the results no longer changed. They are shown in Fig. 3, from which the smooth structure can be seen. (An experiment with a noisy first guess, among others, was made. It began to develop toward the fields in Figs. 3. During the computations, however, a secondary minimum was found. This is not studied here in more detail because of the noisy appearance of the field.)

In the case of a negative tendency, the resulting $q$ values are very negative over the Pacific and the A.tlantic, exceeding $-1.1 q_{0}$ (Fig. 3a). Because negative values intensify the development, barotropic tendencies are intensified over those areas, as would be expected. In addition to the negative extrema, there are two continental ones with positive values.

In the case of a positive tendency (Fig. 3b), the $q_{+}$ values systematically weaken the tendencies over the oceans. Thus, both $q$ fields make the tendencies more negative over the Atlantic and the Pacific, while over North America and Siberia they tend to make the tendencies more positive. Qualitatively this could be summarized by saying that the double-valued $q$ term performs as if the left-hand side of (11) were of the form

$$
\nabla^{2} \frac{\partial \psi}{\partial t}-\frac{q}{\mu}\left|\frac{\partial \psi}{\partial t}\right|
$$

where the single-valued $q \approx q_{+}$.
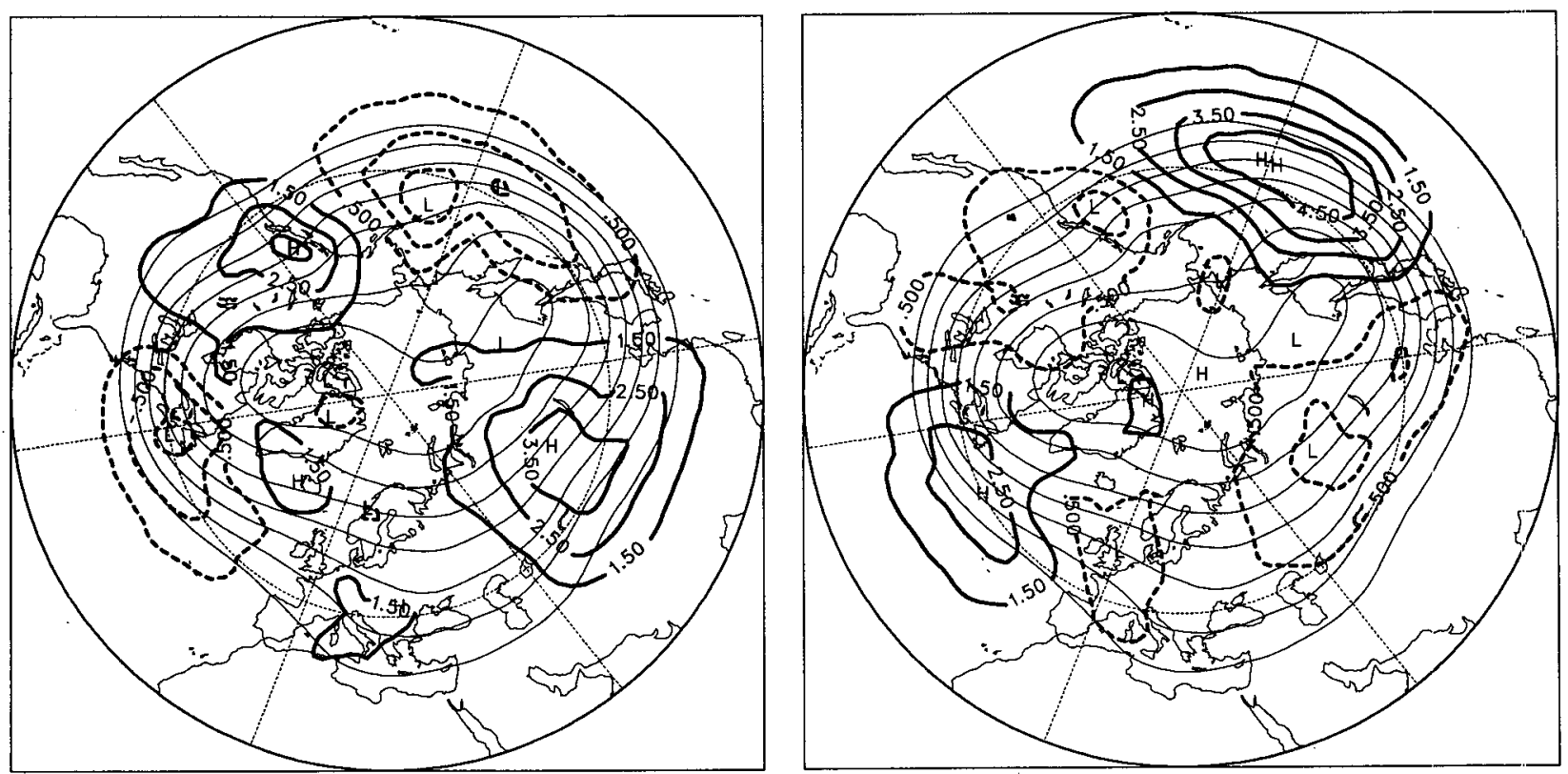

FIG. 3. The double-valued $q$ fields in relative terms of $q / q_{0}$. For negative [positive] tendencies, values of (a) [b] should be applied. The mean $500-\mathrm{hPa}$ height field of the dependent data is shown with thin isolines of $80-\mathrm{m}$ spacing. 
It is clear from Table 1 that the double-valued $q$ fields are more efficient in reducing the forecasting error than the single-valued one (Table 1, third and fourth rows). Note that negative values for the Cressman term are somewhat exceptional, though the positive maxima in Figs. 3 are dominant.

We next try to relate the result to the quasi-stationary waves of the mean circulation. It is implied here that the free long waves move westward and that there are areas of negative (positive) tendencies on the western side of troughs (ridges) in these waves. Further, $q>0$ makes the model divergent, and the long waves are slowed down. When $q<0$, the waves are accelerated. (This assumption is true at least for single waves when $q$ is close to 0 .) We study the effect of $q_{+}, q_{-}$on the movement of free waves over the areas of the standing waves in the mean circulation. In the case of a standing trough, there are four alternatives, with a trough or a ridge approaching or leaving the area of the standing wave. The effects can be summarized as follows.

1) If a trough with preceding negative tendencies is approaching the areas of the standing troughs over the Pacific or the Atlantic, the free wave trough is accelerated toward such an area (Fig. 3a for negative tendencies, $q<0$ on the eastern side of the standing troughs). Thus the standing trough will be intensified.

2) If a trough in the free wave is located over one of the areas of the standing troughs, its movement will be slowed down (Fig. 3a for negative tendencies, $q>0$ on the western side of the standing troughs).

3 ) If a ridge is approaching one of the areas of the standing troughs, it is decelerated (Fig. $3 \mathrm{~b}$ for positive tendencies, $q>0$ on the eastern side of standing troughs).

4) If a ridge of a free wave is leaving one of the areas of the standing troughs, its movement will be accelerated (Fig. $3 b$ for positive tendencies, $q<0$ on the western side of standing troughs).

Thus, the movement of free waves will in all cases maintain or intensify the standing troughs in the mean circulation.

In the case of standing ridges, the extrema in Figs. $3 a, b$ are located over them. Because of this, a more straightforward interpretation can be given in terms of tendencies. For instance, the task of the field in Fig. 3a over the Rocky Mountains is to weaken negative tendencies and therefore prevent any deepening over the area of the standing ridge. Positive tendencies are intensified (Fig. 3b). As a whole, the ridges of the main circulation will be maintained in all cases.

\section{Concluding remarks}

The most surprising feature of the present results is that they do not correspond to the basic theory generally accepted and repeated for decades. The values of $q$ are related neither to $f$ nor to $f^{2}$ as expected. In particular, the double-valued $q$ shows unexpected neg- ative values over large areas. There are no obvious connections with surface wind. The present $q$ fields cannot easily be characterized by saying that they decrease the speed of the long waves because the effect varies locally. Over the Atlantic and Pacific, the wave speeds are effectively damped in the south, whereas in the north, the model is closer to the nondivergent model (cf. Fig. 1). In the case of the double-valued $q$, the speed varies depending on the sign of the tendency and its location. The approximate form found for the Cressman term in the model, $q|\partial \psi / \partial t|$, best tells how far we are from theory.

From the practical point of view, our form of the Cressman term would obviously not have made the daily forecasts much better. Perhaps in some cases the limited memory of computers would have prevented the use of our $q$ field. The systematic error, however, has decreased in such a manner (Table 1) that the present results obviously would have been of interest and would have led to application attempts.

Our results explain why the theoretical values have never been used. Application of an $f^{2}$ dependence would lead even further from the present values. It has been better to use a constant instead. Obviously $q_{0}$ has been a good choice. From Table 1 it can be seen that any reasonable choice works when compared with the nondivergent model.

Why have values like those in Figs. 1 and 3 never been presented before? The present computations would of course have been impossible because of the unknown method and the computational requirements. The structure of the $q$ fields with their few extrema is so simple, however, that it should be possible to recover them using other methods. The term has been important, which should have encouraged study of it.

We suggest that development has been stifled by tradition, in which there has been a concentration on the divergence term and zonal or global means. Further, it may have been difficult to cast doubts upon a theory developed by such renowned meteorologists.

A new feature found is that $q$ may become negative. Nevertheless, the forecasting model performs well, which is obviously due to the fact that $q$ may be negative only locally.

The new framework can be characterized in several different ways. The simplest way is to see $q$ as a multiplier of the tendency. Another way is to see $q$ as a corrector of the systematic error. The $q$ fields can be associated with areas of baroclinity, too. The maxima in Fig. 1 are located, at least partly, eastward of the main areas of baroclinity (cf. Hoskins and Valdes 1990). Thus, over those maxima, baroclinic development can be expected to be most intense. The previously computed illustrative value of $q=3.4 q_{0}$ for a baroclinic case fits well with values over the Pacific in Fig. 1. In this interpretation, the task of $q$ is to parameterize the development due to the baroclinity. The situation is perhaps not that simple, however, because 
similar patterns are found in studies of other subjects. The systematic error in 30-day ECMWF forecasts resembles ours (Palmer et al. 1990). Perhaps we have been working with a type of error that still appears to some extent in the error fields of even the most modern models!

Classically, the Cressman term has been associated with the divergence of the long waves, and its effect is to make the movement of those waves slower. This can be extended to the present results by concluding that $q$ is found especially to control standing long waves because large values in Fig. 1 are either located slightly eastward of the troughs (Atlantic, Pacific) or over ridges (North America, Siberia) in the mean circulation. The weaker maxima around the Black Sea are similarly connected to a standing trough. Thus, the task of the Cressman parameter in the present form would be to maintain the standing waves of the mean circulation. The free waves of the model are affected, too, as soon as they move into areas of large $q$. In section 5, this kind of explanation was found to fit with the fields in Fig. 3.

The previous idea was tested by applying the present technique with the mean field of the dependent data as the only initial analysis. The aim was to find the best parameter field that, when making a forecast starting from the mean field, resulted in the smallest changes within $24 \mathrm{~h}$, thus tending to maintain the mean field. The form of the parameter field, that is, the location and the relative strength of the extrema, was very similar to that in Fig. 1. The result thus supports the idea.

Hence, the fields in Fig. 1 and especially those in Fig. 3 seem to be suitable to maintain the troughs and ridges in the mean circulation. In addition to this, the free waves are also affected. The original Cressman parameter works similarly everywhere without taking into account the features of the main circulation. It can thus be seen as a crude though effective approximation to the present parameter fields.

The extended experiments in section 5 resulted in a form for a model that is difficult to explain physically and is in disagreement with theory. In all, we have three alternatives for the choice: the classical $q$, that in Fig. 1, and that in Fig. 3. The choice depends on the decision maker. Perhaps the classical theory, though in reality approximative, is felt to be the most important factor. For instance, the decision maker may require that $q$ be positive; then the classical $q$ is the only choice. This implies that one has to explain why theory, however, is not fully followed-for example, why $q$ is not related to the Coriolis parameter. A compromise would be to repeat the present computations with the additional constraint of $q>0$. In that case, too, theory is more important than the quality of the forecasts.

Perhaps one can accept minor deviations from theory, in which case Fig. 1 would be the best choice. All deviations from theory, such as the rejection of the dependency between $q$ and $f$, would then have to be explained as minor ones.
Perhaps, in the opinion of the decision maker, the most important factor is the quality of the forecasts. Then Fig. 3 could be accepted, although the corresponding model is far from the classical one. In support of this alternative, it can be said that it is necessary to maintain the mean circulation.

Starting from three different formulations, that is, $q$ is constant, $q$ is given at every grid point, and $q$ is given separately for positive and negative tendencies, we have found three different solutions. The two latter cases are rather similar in terms of the forecast quality. The resulting models clearly differ physically. In this sense the result of the optimization is not unique: as with rather small changes in the initial formulation of the problem, different results can be found.

\section{APPENDIX}

\section{The Consistency of the Tangent Linear-Adjoint Pair}

\section{a. The tangent linear equation and its adjoint in the grid}

As the derivation of the equations is similar to that in Talagrand and Courtier (1987), it will not be repeated here; only the differences due to the numerical approximations in the grid will be dealt with. The time stepping will not be considered. Consequently, we write the equations for one time step. Instead of deriving the equations, we will follow a reversed order and study whether our equations form an adjoint pair.

Talagrand and Courtier [1987, 1314-1315, their Eqs. (1) and (3)] formally apply the adjointness as follows:

$$
\delta J=\left\langle\nabla_{\nu} J, \delta \mathbf{v}\right\rangle=\left\langle G^{*} \nabla_{\nu} J, \delta \mathbf{u}\right\rangle .
$$

Here $\delta J$ is the variation in the cost function, and $\mathbf{v}$ and $\mathbf{u}$ are the output and input of the tangent linear equation $\left(G^{\prime}\right)$, the adjoint of which is $G^{*}$. Applying our definition of the inner product [cf. (2)], (A1) becomes

$$
\begin{aligned}
\delta P & =-\sum \delta \psi^{\prime}\left(\nabla^{2}-\frac{q}{\mu}\right) G^{\prime} \delta \psi \\
& =-\sum G^{\prime * \delta \psi^{\prime}}\left(\nabla^{2}-\frac{q}{\mu}\right) \delta \psi .
\end{aligned}
$$

Here $\delta P, \delta \psi, G^{\prime} \delta \psi, \delta \psi^{\prime}$, and $G^{*} \delta \psi^{\prime}$ correspond to $\delta J$, $\delta \mathbf{u}, \delta \mathbf{v}, \nabla_{v} J$, and $G^{\prime *} \nabla_{v} J$, respectively. The output of the tangent linear equation ( $1 b$ ) during one time step is

$$
\begin{aligned}
G^{\prime} \delta \psi=\left(\nabla^{2}-\frac{q}{\mu}\right)^{-1}\left[J\left(\mu \nabla^{2} \psi+f, \delta \psi\right)\right. \\
\left.+J\left(\mu \nabla^{2} \delta \psi, \psi\right)\right] 2 \Delta t
\end{aligned}
$$

or symbolically

$$
G^{\prime} \delta \psi=\left(\nabla^{2}-\frac{q}{\mu}\right)^{-1}\left(J_{1}+J_{2}\right) 2 \Delta t .
$$


For the output of the adjoint equation, we have found [cf. (3)]

$$
\begin{aligned}
G^{*} \delta \psi^{\prime}=-\left(\nabla^{2}-\frac{q}{\mu}\right)^{-1}\left[-J^{*}\left(\delta \psi^{\prime}, \mu \nabla^{2} \psi+f^{\prime}\right)\right. & \\
& \left.+\nabla^{2} \mu J^{*}\left(\delta \psi^{\prime}, \psi\right)\right] 2 \Delta t,
\end{aligned}
$$

or symbolically

$$
G^{*} \delta \psi^{\prime}=-\left(\nabla^{2}-\frac{q}{\mu}\right)^{-1}\left(J_{1}^{*}+J_{2}^{*}\right) 2 \Delta t .
$$

Replacing these in (A2), one gets

$$
\delta P=-\sum \delta \psi^{\prime}\left(J_{1}+J_{2}\right) 2 \Delta t
$$

and

$\delta P=\sum\left(\nabla^{2}-\frac{q}{\mu}\right)^{-1}\left[\left(J_{1}^{*}+J_{2}^{*}\right) 2 \Delta t\right]\left(\nabla^{2}-\frac{q}{\mu}\right) \delta \psi$.

Thus far, the use of the equations has not differed from the continuous case. In the following, the numerical approximations are used. The following equivalence can be shown to be true by first writing out the sums and then comparing both sides term by term:

$$
\sum_{i, j} a_{i, j}\left(\nabla^{2}-\frac{q_{i, j}}{\mu_{i, j}}\right) b_{i, j}=\sum_{i, j} b_{i, j}\left(\nabla^{2}-\frac{q_{i, j}}{\mu_{i, j}}\right) a_{i, j} .
$$

It is implied that $a$ and $b$ vanish at the boundaries. Now (A7) can be written

$$
\delta P=\sum \delta \psi\left(J_{1}^{*}+J_{2}^{*}\right) 2 \Delta t .
$$

For (A2) to be valid, it is necessary that (A6) and (A8) result in equal values of $\delta P$. Thus,

$$
-\sum \delta \psi^{\prime}\left(J_{1}+J_{2}\right) 2 \Delta t=\sum \delta \psi\left(J_{1}^{*}+J_{2}^{*}\right) 2 \Delta t .
$$

This is shown to hold by writing out [making use of (4)], the sums and symbols. Then both sides are compared term by term and found to be equal. Thus, (A2) holds and our equations form an adjoint pair.

\section{$b$. The variation with respect to $q$}

The tangent linear equation ( $1 \mathrm{~b}$ ) is derived from (1a). By taking into account the variation with respect to $q$,

$$
\tilde{G}^{\prime} \delta \psi=\left(\nabla^{2}-\frac{q}{\mu}\right)^{-1}\left[\left(J_{1}+J_{2}\right) 2 \Delta t+\frac{\partial \psi}{\partial t} \frac{\delta q}{\mu}\right]
$$

is obtained, instead of (A4). Instead of (A6), we will have

$$
\delta P=-\sum \delta \psi^{\prime}\left(J_{1}+J_{2}\right) 2 \Delta t-\sum \delta \psi^{\prime} \frac{\partial \psi}{\partial t} \frac{\delta q}{\mu} .
$$

Thus, a variation of $\delta q$ causes a variation in $P$ of

$$
\delta P=-\delta \psi^{\prime} \frac{\delta q}{\mu} \frac{\partial \psi}{\partial t} .
$$

The variation in $P$ with respect to $q$ will be found by first performing one forecast-adjoint cycle with (1a) and (3) to produce $\partial \psi / \partial t$ and $\delta \psi^{\prime}$. Then (6) is applied. This is the form that (A10) takes when the time stepping is studied.

\section{REFERENCES}

Amodei, L., and P. Bernardet, 1990: Adjoint assimilation technique applied to initial-boundary value problem. Int. Symp. on Assimilation of Observations in Meteorology and Oceanography, WMO, Clermont-Ferrand, France, 482-485.

Bolin, B., 1956: An improved barotropic model and some aspects of using the balance equation for three dimensional flow. Tellus, $8,61-75$

Charney, J. G., 1973: Planetary fluid dynamics. Dynamic Meteorology, P. Morel, Ed., Reidel, 97-351.

Courtier, P., and O. Talagrand, 1987: Variational assimilation of meteorological observations with the adjoint vorticity equation. II: Numerical results. Quart. J. Roy. Meteor. Soc., 113, 13291347.

Cressman, G. P., 1958: Barotropic divergence and very long atmospheric waves. Mon. Wea. Rev., 86, 293-297.

Döös, B. R., 1969: Numerical weather forecasting with the barotropic model. Lectures on Numerical Short-range Weather Prediction, V. V. Bykov, Ed., WMO, Gidrometeoizdat, 188-209.

Haltiner, G. J., and R. T. Williams, 1990: Numerical Prediction and Dynamic Meteorology, 2d ed., Wiley and Sons, $477 \mathrm{pp}$.

Holton, J. R., 1979: An Introduction to Dynamic Meteorology, 2d ed., Academic Press, $391 \mathrm{pp}$.

Hoskins, B. J., and P. J. Valdes, 1990: On the existence of stormtracks. J. Atmos. Sci., 47, 1854-1864.

Palmer, T. N., C. Brankovic, F. Molteni, and S. Tibaldi, 1990: Extended-range predictions with ECMWF models: Interannual variability in operational model integrations. Quart. J. Roy. Meteor. Soc., 116, 799-834.

Rossby, C.-G., and collaborators, 1939: Relation between variations in the intensity of the zonal circulation of the atmosphere and the displacements of the semi-permanent centers of action. $J$. Mar. Res., 2, 38-55.

Talagrand, O., and P. Courtier, 1987: Variational assimilation of meteorological observations with the adjoint vorticity equation. I: Theory. Quart. J. Roy. Meteor. Soc., 113, 1311-1328.

Thacker, W. C., 1988: Three lectures on fitting numerical models to observations. Rep. No. GKSS 87/E/65, GKSS-Forschungszentrum, Geesthacht, Germany, $64 \mathrm{pp}$.

Wiin-Nielsen, A., 1991: The birth of numerical weather prediction. Tellus, 43AB, 36-52.

Wolff, P. M., 1958: The error in numerical forecasts due to retrogression of ultra-long waves. Mon. Wea. Rev., 86, 245-250. 